\title{
White blood cell count to mean platelet volume ratio: A novel and promising prognostic marker for ST-segment elevation myocardial infarction
}

\author{
Gökhan Çiçek ${ }^{1}$, Sadık Kadri Açıkgöz ${ }^{2}$, Çağrı Yayla ${ }^{2}$, Harun Kundi ${ }^{1}$, Mehmet İleri $^{1}$ \\ ${ }^{1}$ Department of Cardiology, Ankara Numune Education and Research Hospital, Ankara, Turkey \\ ${ }^{2}$ Department of Cardiology, Turkiye Yuksek Ihtisas Education and Research Hospital, Ankara, Turkey
}

\begin{abstract}
Background: Increased white blood cell (WBC) count is associated with increased mortality in patients with ST-segment elevation myocardial infarction (STEMI). We aimed to evaluate predictive value of admission WBC to mean platelet volume (MPV) ratio (WMR) on prognosis in patients undergoing primary percutaneous coronary intervention (
\end{abstract}

Methods: A total of 2,603 consecutive patients with STEMI who underwent PPCI were recruited for the study. Follow-up data were obtained from digital records, patient files or by telephone interview with patients, family members, or primary care physicians.

Results: WMR has the highest area under receiver operating characteristic (ROC) curve and pairwise comparisons of the ROC curves revealed that WMR has the higher discriminative ability for long-term mortality than WBC, MPV, red blood cell distribution with (RDW), WBC-MPV combination, and platelet to lymphocyte ratio and neutrophil to lymphocyte ratio (PLR-NLR) combination in patients undergoing pPCI for STEMI (a WMR value of 1,653.47 was also found as threshold value for mortality with $75.4 \%$ sensitivity and $87.3 \%$ specificity by ROC curve analysis).

Conclusions: Higher WMR value on admission was associated with worse outcomes in patients with STEMI and independently better predicted the long-term mortality than other complete blood count components, such as MPV, RDW, PLR-NLR and WBC-MPV combinations. (Cardiol J 2016; 23, 3: 225-235)

Key words: mean platelet volume, ST-segment elevation myocardial infarction, white blood cell

\section{Introduction}

The role of inflammation in coronary artery disease (CAD) has been widely recognized [1]. White blood cells (WBCs) and platelets have potential roles in the pathogenesis of ST-segment elevation myocardial infarction (STEMI) [2, 3]. Increased WBC count is associated with increased mortality in patients with STEMI [4]. Mean platelet volume (MPV) is a potentially useful biomarker of platelet activity [5]. Apart from WBC count, other blood count parameters such as neutrophil count, red blood cell distribution with (RDW), mean platelet volume (MPV), high neutrophil to lymphocyte ratio (NLR) and high platelet to lymphocyte ratio (PLR) also seem to have prognostic value in STEMI [6-10]. As a combination of both WBC and mean platelet volume, WBC count to mean

Address for correspondence: Gökhan Çiçek, MD, Talatpasa Bulvari, 06100, Sihhiye, Ankara, Turkey, tel: +90 31250840 00, fax: +90 31231034 60, e-mail: drvaristor@hotmail.com; drvaristor@gmail.com 
platelet volume ratio (WMR) has been recently found as a novel non-invasive marker predicting long-term outcomes in patients with non-ST elevation myocardial infarction (NSTEMI) [11]. Until now, the use of this marker for cardiovascular (CV) prognosis has not been investigated in patients undergoing primary percutaneous coronary intervention (pPCI) for STEMI. Therefore, we evaluated whether admission WMR obtains considerable prognostic information in patients undergoing pPCI for STEMI in a large population. We also assessed if this novel marker can provide further information in addition to other blood cell count parameters.

\section{Methods}

\section{Study design}

A total of 2,603 consecutive patients with STEMI who presented within $12 \mathrm{~h}$ from the onset of symptoms and underwent $\mathrm{pPCI}$ were recruited for the study. The study was approved by the Local Ethics Committee and all subjects gave their written informed consent. Patients with active infection, autoimmune diseases, hematologic proliferative disease, malignant neoplasia, and other chronic systemic disease were excluded from the study. Symptoms of myocardial ischemia and ST-segment elevation $\geq 1 \mathrm{~mm}$ in two contiguous electrocardiographic leads or new onset of complete left bundle-branch block were defined as ST elevation myocardial infarction. A previous diagnosis of diabetes mellitus (DM), use of antidiabetic medicines, or a fasting venous blood glucose level of $126 \mathrm{mg} / \mathrm{dL}$ on two occasions in previously untreated patients were required for a diagnosis of DM. Hypertension (HT) was defined as a previous use of antihypertensive medications, a systolic pressure higher than $140 \mathrm{~mm} \mathrm{Hg}$, or a diastolic pressure higher than $90 \mathrm{~mm} \mathrm{Hg}$ on at least two separate measurements. Hypercholesterolemia was defined as total cholesterol of at least $200 \mathrm{mg} / \mathrm{dL}$. The glomerular filtration rate (GFR) was estimated by using the Modification of Diet in Renal Disease (MDRD) equation at admission. Left ventricular ejection fraction was assessed by modified biplane Simpson's method in two-dimensional (2D) echocardiography.

Definition of re-infarction was formed according to the Third Universal Definition of Myocardial Infarction [12]. Target vessel revascularization (TVR) was defined as the need for PCI or coronary surgery because of restenosis or re-occlusion of the infarct-related artery (IRA). Major adverse cardiac events (MACE) were defined as CV death, re-infarction, or TVR.

\section{Coronary angiography}

All pPCI procedures were performed after loading dose of $300 \mathrm{mg}$ acetylsalicylic acid and $300 \mathrm{mg}$ clopidogrel by experienced interventional cardiologists who were unaware of the study. Coronary angiography was performed using the percutaneous femoral route. Heparin $(100 \mathrm{U} / \mathrm{kg})$ was administered when the coronary anatomy was first assessed and the use of glycoprotein IIb/IIIa inhibitors was left to the preference of the operator. Follow-up data were obtained from digital records, patient files or by telephone interview with patients, family members, or primary care physicians.

\section{Laboratory measurements}

Venous blood samples were obtained from all patients for hematologic and biochemical measurements on admission. An automated complete blood count using a Coulter LH 780 Hematology Analyzer (Beckman Coulter Ireland Inc., Mervue, Galway, Ireland) was used to measure hematologic parameters. The PLR was calculated as the ratio of the platelets and lymphocytes, the NLR was calculated as the ratio of the neutrophils and lymphocytes, and WMR was calculated as the ratio of WBC count and MPV, all obtained from the same automated blood sample at admission of the study. The 12-h fasting serum lipid profile was measured by standard enzymatic methods.

\section{Statistical analysis}

Analyses were performed using SPSS Statistics, version 20.0 (IBM SPSS Inc., Chicago, IL) and Medcalc 11.4.2 (MedCalc Software, Mariakerke, Belgium). Continuous variables were tested for normal distribution by Kolmogorov-Smirnov test and expressed as mean \pm standard deviation or median (interquartile range $[\mathrm{IQR}]$ ) values as appropriate. One way ANOVA was used to compare data with normal distribution and the KruskalWallis $\mathrm{H}$ test was applied to compare the data without normal distribution and significance after Bonferroni correction for multiple comparisons. Categorical variables were expressed as numbers and percentiles and compared by $\chi^{2}$ and Fisher's exact tests. Among parameters that are found to be univariable and associated with the outcome, but also in a strong relationship with some others; only the variables showing the strongest univariable association with the outcome $(\mathrm{p}<0.25)$ 
are included in the stepwise multivariable Cox regression analysis. Since there is a high correlation (Goodman and Kruskal's Gamma Correlation, $\mathrm{G}=$ $=0.976, \mathrm{p}<0.001)$ which can cause multicollinearity between WMR risk groups and WBC-MPV combination risk groups, WMR and WBC-MPV combinations were included in two distinct Cox-regression models. Kaplan-Meier survival curves and the log-rank test were used to compare all-cause mortality between the low, intermediate, and high-risk groups. A 2-sided p value $<0.05$ was considered statistically significant.

\section{Results}

In total, 2,603 patients, $2,129(81.8 \%)$ males and $474(18.2 \%)$ females, were recruited for the study. Mean age of the participants was $57.6 \pm$ \pm 11.8. Among the participants, $625(24.0 \%)$ had DM, 1,085 (41.7\%) had HT and 834 (32.0\%) had hyperlipidemia. Median follow-up time was 12 (1-54) months.

Cutoff values for PLR, NLR, WBC, MPV and WMR were calculated with receiver-operating characteristics (ROC) curves and risk stratification was made according to WBC-MPV combination and WMR values. A PLR of 162.30 (50.8\% sensitivity, $69.9 \%$ specificity), a NLR of 6.32 (53.3\% sensitivity, $75.5 \%$ specificity), a WBC of 14,400 (57.8\% sensitivity, $90.3 \%$ specificity) and a MPV of 8.1 (73.2\% sensitivity, $68.0 \%$ specificity) were found as threshold values for mortality by ROC curves. If both PLR and NLR were above the selected threshold values, patients were classified as "high-risk". If either PLR or NLR were above the threshold individually, patients were classified as "intermediaterisk". If both levels were under threshold values, patients were classified as "low-risk". In the second model, if both WBC and MPV were above the selected threshold values, patients were classified as "high-risk". If either WBC or MPV were above the threshold individually, patients were classified as "intermediate-risk". If both levels were under threshold values, patients were classified as "lowrisk". A WMR value of $1,653.47$ was also found as threshold value for mortality with $75.4 \%$ sensitivity and $87.3 \%$ specificity by ROC curve analysis. A second ROC curve analysis was conducted for patients with WMR $>1,653.47$ and a WMR value of $1,824.18$ was found to show mortality with $77 \%$ sensitivity and $100 \%$ specificity.

Mean age and rates of female gender, DM, $\mathrm{HT}$, anterior myocardial infarction, and cardiogenic shock were higher in both high-risk groups compared to low and medium risk groups (Table 1).
Laboratory findings of the patients were summarized in Table 2. In both high-risk groups, creatinine, peak creatinine kinase-MB, glucose, RDW, WBC, PLR and NLR were higher and hemoglobin, MPV, triglyceride and GFR were lower.

Distribution of culprit vessels, number of diseased vessels, stent length, stent diameter and tirofiban use were similar among risk groups. However, stent use was less frequent in both high-risk groups compared to others. Patients with post-procedural Thrombolysis in Myocardial Infarction (TIMI) 3 flow were less frequent in high-risk groups (Table 3 ).

In-hospital and long-term CV events were shown in Table 4. Rates of in-hospital mortality, MACE, stroke, ventricular tachycardia-fibrillation, heart failure, cardiogenic shock, atrial fibrillation, temporary pacemaker use, gastrointestinal bleeding, need for hemodialysis and blood transfusion were significantly higher in both high-risk groups. Rate of in-hospital re-infarction was higher in WMR high-risk group but did not differ between WBC-MPV combination risk groups. Rates of long-term $\mathrm{CV}$ mortality, MACE, heart failure and re-infarction was significantly higher in both high risk groups compared to low and intermediate-risk groups. However, long-term stroke and TVR rates were similar among the groups.

Age $>70$, male gender, HT, DM, Killip class $>1$, heart rate $>100 \mathrm{bpm}$, admission anemia, RDW and PLR-NLR combination were found as independent predictors of long-term CV mortality in Cox regression models I and II. In addition, WBC-MPV combination was an independent predictor of mortality in model I and WBC/PLR was an independent predictor of mortality in model II. Strongest predictors of mortality were WBC-MPV combination high-risk group in model I and WMR high-risk group in model II. Since the -2 Log Likelihood (LL) value was lower in model II than model I (model I: $-2 \mathrm{LL}=2,675.6, \chi^{2}=635.7, \mathrm{p}<0.001$; model II: $\left.2 \mathrm{LL}=2,564.8, \chi^{2}=811.5, \mathrm{p}<0.001\right)$, model II was found more appropriate for mortality prediction (Table 5).

Receiver operating characteristic curves of WBC-MPV combination, WMR, PLR-NLR combination and their components for long-term mortality is shown in Figure 1. WMR has the highest area under ROC curve and pairwise comparisons of the ROC curves revealed that WMR has the highest discriminative ability for long-term mortality (Fig. 1). A log-rank p value $<0.001$ was obtained while comparing survival amongst three risk groups in both risk models (Fig. 2). 
Table 1. Baseline characteristics of the patients.

\begin{tabular}{|c|c|c|c|c|c|c|c|c|}
\hline \multirow[t]{2}{*}{ Variables } & \multicolumn{3}{|c|}{ Combined WBC-MPV } & \multirow[t]{2}{*}{$\mathbf{P}$} & \multicolumn{3}{|c|}{ WMR } & \multirow[t]{2}{*}{$\mathbf{P}$} \\
\hline & $\begin{array}{l}\text { Low risk } \\
(n=1,209)\end{array}$ & $\begin{array}{c}\text { Intermediate } \\
\text { risk } \\
(n=1,266)\end{array}$ & $\begin{array}{l}\text { High risk } \\
(\mathrm{n}=128)\end{array}$ & & $\begin{array}{c}\text { Low risk } \\
(\mathrm{n}=2,120)\end{array}$ & $\begin{array}{c}\text { Intermediate } \\
\text { risk } \\
(n=340)\end{array}$ & $\begin{array}{l}\text { High risk } \\
\text { (n=143) }\end{array}$ & \\
\hline Age [years] & $57.5 \pm 11.4$ & $57.2 \pm 12$ & $62.3 \pm 12^{\ddagger \ddagger}$ & $<0.001^{*}$ & $57.6 \pm 11.7$ & $55.1 \pm 11.7$ & $63.9 \pm 11.8^{\dagger \ddagger}$ & $<0.001^{*}$ \\
\hline Male gender & $978(80.9)$ & $1062(83.9)$ & $89(69.5)$ & $0.001 *$ & $1738(82)$ & $292(85.9)$ & $99(69.2)$ & $<0.001^{*}$ \\
\hline Smoking & $730(60.4)$ & $757(59.8)$ & $70(54.7)$ & 0.458 & $1251(59)$ & $233(68.5)$ & $73(51)$ & $<0.001^{*}$ \\
\hline Diabetes & $294(24.3)$ & $274(21.6)$ & $57(44.5)$ & $<0.001 *$ & * $486(22.9)$ & $71(20.9)$ & $68(47.6)$ & $<0.001^{*}$ \\
\hline Hypertension & 507 (41.9) & $511(40.4)$ & $67(52.3)$ & $0.033^{*}$ & $875(41.3)$ & $136(40)$ & $74(51.7)$ & $0.039 *$ \\
\hline Family history & $237(19.6)$ & $258(20.4)$ & $12(9.4)$ & $0.007^{*}$ & $416(19.6)$ & $78(22.9)$ & $13(9.1)$ & $0.001 *$ \\
\hline Hyperlipidemia & $393(32.5)$ & $410(32.4)$ & $31(24.2)$ & 0.147 & $693(32.7)$ & $109(32.1)$ & $32(22.4)$ & 0.138 \\
\hline Dialysis & $0(0)$ & $4(0.3)$ & $1(0.8)$ & 0.082 & $2(0.1)$ & $2(0.6)$ & $1(0.7)$ & 0.065 \\
\hline Prior CABG & $45(3.7)$ & $32(2.5)$ & $5(3.9)$ & 0.178 & $74(3.5)$ & $3(0.9)$ & $5(3.5)$ & $0.018^{*}$ \\
\hline $\mathrm{PCl}$ history & $107(8.9)$ & $109(8.6)$ & $19(14.8)$ & $0.042^{*}$ & $181(8.5)$ & $29(8.5)$ & $25(17.5)$ & $0.003^{*}$ \\
\hline Prior Ml & $152(12.6)$ & $129(10.2)$ & $24(18.8)$ & $0.008^{*}$ & $241(11.4)$ & $32(9.4)$ & $32(22.4)$ & $0.001 *$ \\
\hline Anterior $\mathrm{MI}$ & $527(43.6)$ & $608(48)$ & $75(58.6)$ & $0.002 *$ & $937(44.2)$ & $189(55.6)$ & $84(58.7)$ & $<0.001^{*}$ \\
\hline Admission CS & $15(1.2)$ & $32(2.5)$ & $30(23.4)$ & $<0.001^{*}$ & * $\quad 29(1.4)$ & $15(4.4)$ & $33(23.1)$ & $<0.001^{*}$ \\
\hline Angina-to-perfusion time [h] & $4 \pm 118$ & $4 \pm 58$ & $4.5 \pm 10$ & 0.462 & $4 \pm 88$ & $3.5 \pm 58$ & $4 \pm 8$ & 0.977 \\
\hline Killip class $>1$ & $47(3.9)$ & $60(4.7)$ & $50(39.1)$ & $<0.001^{*}$ & * $74(3.5)$ & $29(8.5)$ & $54(37.8)$ & $<0.001^{*}$ \\
\hline $\mathrm{SBP}<100 \mathrm{~mm} \mathrm{Hg}$ & $89(7.4)$ & $102(8.1)$ & $44(34.4)$ & $<0.001^{*}$ & $149(7)$ & $34(10)$ & $52(36.4)$ & $<0.001^{*}$ \\
\hline Heart rate $>100 \mathrm{bpm}$ & $34(2.8)$ & $52(4.1)$ & $37(28.9)$ & $<0.001^{*}$ & * $56(2.6)$ & $26(7.6)$ & $41(28.7)$ & $<0.001^{*}$ \\
\hline Admission anemia & $289(23.9)$ & $316(25)$ & $43(33.6)$ & 0.055 & $522(24.6)$ & $70(20.6)$ & $56(39.2)$ & $<0.001^{*}$ \\
\hline LVEF [\%] & $48.2 \pm 7.8$ & $47.3 \pm 7.8$ & $41 \pm 11.3^{\text {tf }}$ & $<0.001 *$ & $48.1 \pm 7.6$ & $46.5 \pm 8.8$ & $40.5 \pm 11.3^{\dagger \neq}$ & $<0.001^{*}$ \\
\hline Time of hospital stay [days] & $6(3)$ & $6(3)$ & $7(8)$ & 0.374 & $6(3)$ & $6(3)$ & $7(8)$ & 0.326 \\
\hline Follow-up period [months] & $9(22)$ & $18(28)^{\dagger}$ & $2(9.8)^{\dagger \ddagger}$ & $<0.001^{*}$ & $13(25)$ & $14(28)$ & $1(9)^{\dagger \neq}$ & $<0.001^{*}$ \\
\hline
\end{tabular}

Continues variables are reported mean \pm standard deviation or median (interquarticle range). Categorical variables are reported $n(\%) .{ }^{*} p<0.05$ was considered significant; $† p<0.05$ vs. low risk (Bonferroni correction); $\neq p<0.05$ vs. intermediate risk (Bonferroni correction); CABG — coronary artery bypass grafting; CS — cardiogenic shock; LVEF — left ventricular ejection fraction; MI — myocardial infarction; SBP — systolic blood pressure; PCl - percutaneous coronary intervention; WBC-MPV - white blood cell-mean platelet volume; WMR - white blood cell count to mean platelet volume ratio

\section{Discussion}

In this study, we have demonstrated for the first time that elevated admission WMR was associated with MACE and worse outcomes during in-hospital and long-term follow-up in patients with STEMI. Age, male gender, HT, anemia, RDW, PLRNLR combination, WBC-MPV combination and WMR were the independent predictors of mortality in patients with STEMI. Moreover, higher WMR was associated with a significant increase in the risk of MACE incidence, and it was a stronger marker than MPV, RDW, PLR-NLR and WBC-MPV combinations in prediction of the in-hospital and long-term clinical outcomes.

Previous studies showed that some basic hematologic parameters such as MPV, RDW, NLR and PLR may have a role in predicting worse outcomes in patients with STEMI undergoing $\mathrm{pPCI}$ $[7,13-15]$. As a constituent of the link between inflammation, thrombosis, and atherogenesis, platelets have a prominent role in progression of atherosclerosis and an increase in the platelet count can show advanced thrombocyte activation and megakaryocytic augmentation. Platelet surface molecules are essential in the interaction with endothelial cells, leukocytes and matrix molecules affecting atherogenesis. Platelets play an active role in platelet-fibrin formation and development of acute myocardial infarction. Several studies have showed that higher platelet count and lymphopenia were related with poor clinical outcomes in various cardiovascular diseases [10, 13]. Azab et al. [16] showed that increased PLR is an independent predictor of long-term mortality in patients with NSTEMI. MPV is a useful marker of platelet activity. Several studies showed close relationship between MPV levels and worse outcomes in patients with acute coronary syndromes (ACS) $[8,17]$. Bigger platelets made from activated mega- 


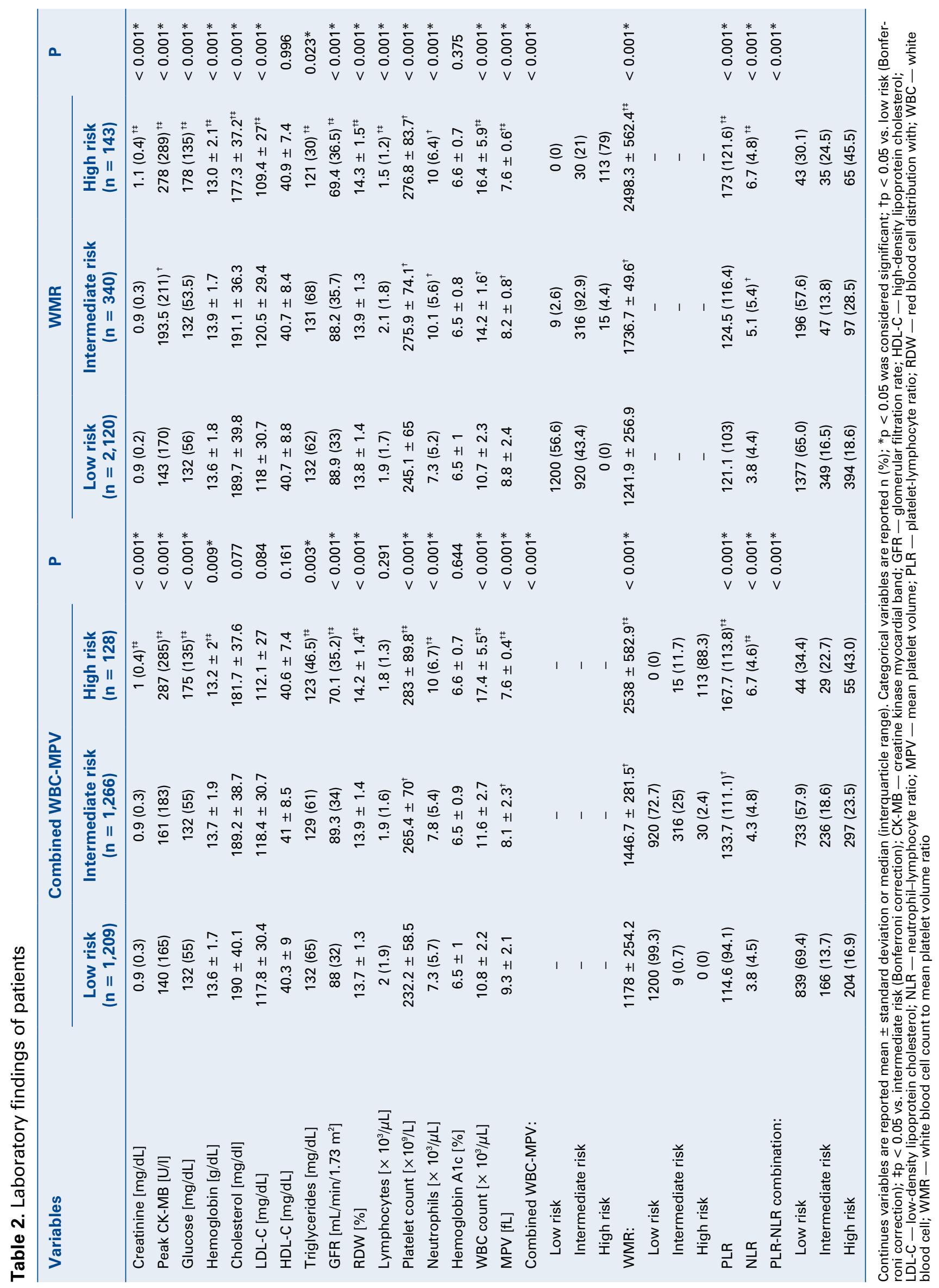




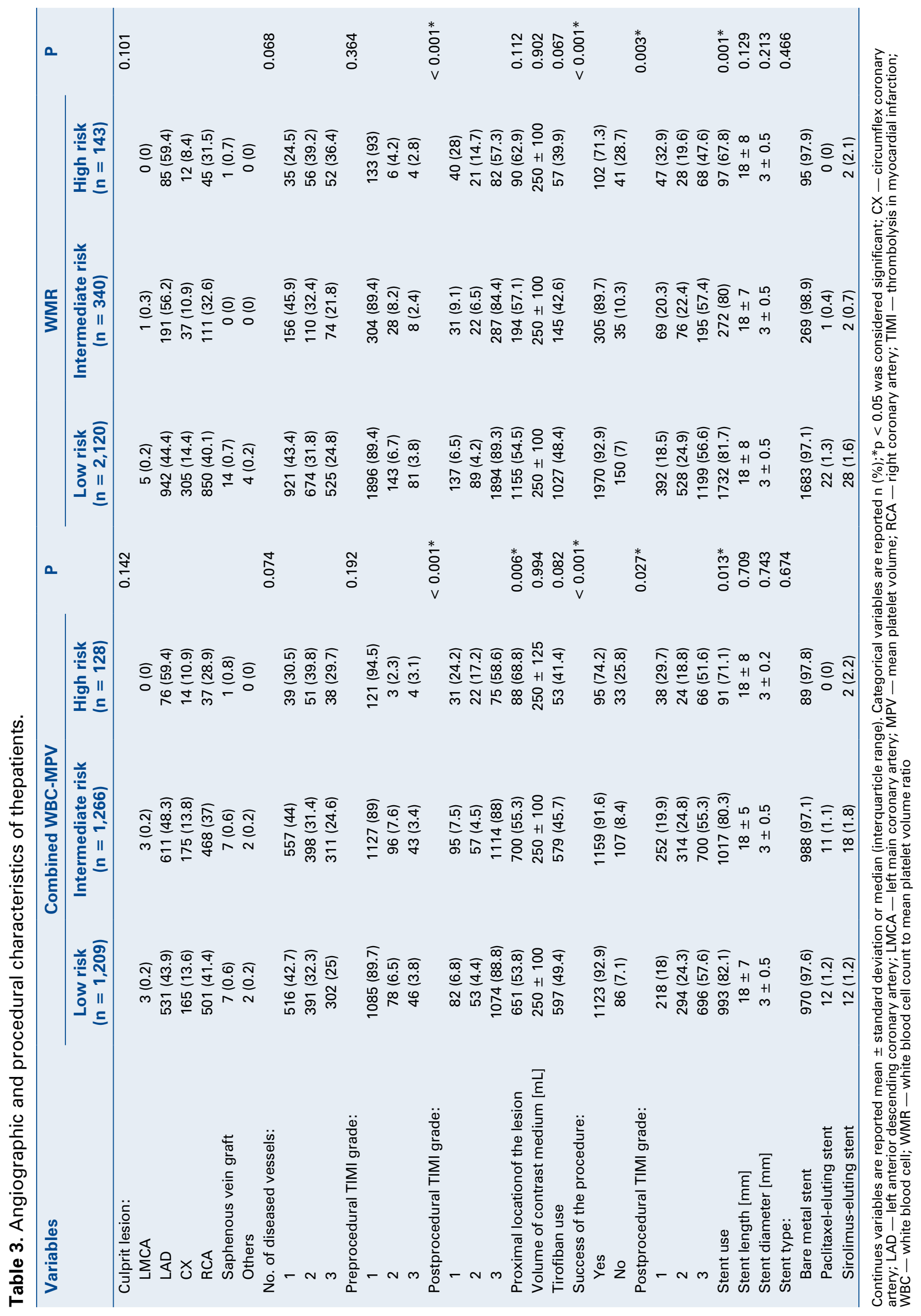


Table 4. In-hospital and long-term cardiac events.

\begin{tabular}{|c|c|c|c|c|c|c|c|c|}
\hline \multirow[t]{2}{*}{ Event } & \multicolumn{3}{|c|}{ Combined WBC-MPV } & \multirow[t]{2}{*}{$\mathbf{P}$} & \multicolumn{3}{|c|}{ WMR } & \multirow[t]{2}{*}{$\mathbf{P}$} \\
\hline & $\begin{array}{c}\text { Low risk } \\
\text { ( } \mathrm{n}=1,209)\end{array}$ & $\begin{array}{c}\text { Intermediate } \\
\text { risk } \\
(n=1,266)\end{array}$ & $\begin{array}{l}\text { High risk } \\
(\mathrm{n}=128)\end{array}$ & & $\begin{array}{l}\text { Low risk } \\
(n=2,120)\end{array}$ & $\begin{array}{c}\text { Intermediate } \\
\text { risk } \\
(n=340)\end{array}$ & $\begin{array}{l}\text { High risk } \\
(\mathrm{n}=143)\end{array}$ & \\
\hline \multicolumn{9}{|c|}{ In-hospital event and complications: } \\
\hline In-hospital mortality & $10(0.8)$ & $37(2.9)$ & $55(43)$ & $<0.001^{*}$ & $24(1.1)$ & $14(4.1)$ & $64(44.8)$ & $<0.001 *$ \\
\hline Reinfarction & $25(2.1)$ & $26(2.1)$ & $6(4.7)$ & 0.153 & $39(1.8)$ & $12(3.5)$ & $6(4.2)$ & $0.031 *$ \\
\hline TVR & $52(4.3)$ & $49(3.9)$ & $9(7)$ & 0.214 & $84(4)$ & $15(4.4)$ & $11(7.7)$ & 0.112 \\
\hline MACE & $59(4.9)$ & $83(6.6)$ & $60(46.9)$ & $<0.001^{*}$ & $104(4.9)$ & $29(8.5)$ & $69(48.3)$ & $<0.001^{*}$ \\
\hline Stroke & $1(0.1)$ & $10(0.8)$ & $5(3.9)$ & $<0.001^{*}$ & $6(0.3)$ & $4(1.2)$ & $6(4.2)$ & $<0.001^{*}$ \\
\hline CPR & $22(1.8)$ & $45(3.6)$ & $56(43.8)$ & $<0.001^{*}$ & $40(1.9)$ & $18(5.3)$ & $65(45.5)$ & $<0.001^{*}$ \\
\hline Hemodialysis & $6(0.5)$ & $8(0.6)$ & $7(5.5)$ & $<0.001^{*}$ & $10(0.5)$ & $3(0.9)$ & $8(5.6)$ & $<0.001^{*}$ \\
\hline VT/VF & $42(3.5)$ & $56(4.4)$ & $39(30.5)$ & $<0.001^{*}$ & $72(3.4)$ & $22(6.5)$ & $43(30.1)$ & $<0.001 *$ \\
\hline Heart failure & $85(7)$ & $139(11)$ & $64(50.0)$ & $<0.001^{*}$ & $172(8.1)$ & $47(13.8)$ & $69(48.3)$ & $<0.001^{*}$ \\
\hline Requaring inotrope & $47(3.9)$ & $89(7)$ & $67(52.3)$ & $<0.001^{*}$ & $99(4.7)$ & $29(8.5)$ & $75(52.4)$ & $<0.001^{*}$ \\
\hline $\begin{array}{l}\text { Cardiogenic shock } \\
\text { and IABP }\end{array}$ & $18(1.5)$ & $37(2.9)$ & $48(37.5)$ & $<0.001^{*}$ & $34(1.6)$ & $17(5)$ & $52(36.4)$ & $<0.001^{*}$ \\
\hline Atrial fibrillation & $20(1.7)$ & $20(1.6)$ & $7(5.5)$ & $0.019^{*}$ & $32(1.5)$ & $7(2.1)$ & $8(5.6)$ & $0.007^{*}$ \\
\hline Complete AVB & $39(3.2)$ & $37(2.9)$ & $20(15.6)$ & $<0.001^{*}$ & $67(3.2)$ & $10(2.9)$ & $19(13.3)$ & $<0.001^{*}$ \\
\hline Transient pacemaker & $41(3.4)$ & $26(2.1)$ & $23(18.0)$ & $<0.001^{*}$ & $59(2.8)$ & $8(2.4)$ & $23(16.1)$ & $<0.001 *$ \\
\hline GI bleeding & $7(0.6)$ & $14(1.1)$ & $5(3.9)$ & $0.008^{*}$ & $15(0.7)$ & $6(1.8)$ & $5(3.5)$ & $0.004^{*}$ \\
\hline Access site complication & $56(4.6)$ & $47(3.7)$ & $7(5.5)$ & 0.367 & $87(4.1)$ & $17(5)$ & $6(4.2)$ & 0.775 \\
\hline Acute stent thrombosis & $16(1.3)$ & $12(0.9)$ & $3(2.3)$ & 0.229 & $26(1.2)$ & $2(0.6)$ & $3(2.1)$ & 0.329 \\
\hline Blood transfusion & $39(3.2)$ & $39(3.1)$ & $13(10.2)$ & $0.001 *$ & $61(2.9)$ & $17(5)$ & $13(9.1)$ & $<0.001^{*}$ \\
\hline \multicolumn{9}{|l|}{ Long-term cardiac events: } \\
\hline Cardiovascular mortality & $14(1.2)$ & $81(6.4)$ & $68(53.1)$ & $<0.001^{*}$ & $42(2)$ & $29(8.5)$ & $92(64.3)$ & $<0.001^{*}$ \\
\hline Heart failure & $58(4.8)$ & $79(6.2)$ & $27(21.1)$ & $<0.001^{*}$ & $106(5)$ & $24(7.1)$ & $34(23.8)$ & $<0.001^{*}$ \\
\hline Stroke & $12(1)$ & $15(1.2)$ & $2(1.6)$ & 0.693 & $24(1.1)$ & $1(0.3)$ & $4(2.8)$ & 0.068 \\
\hline Reinfarction & $50(4.1)$ & $90(7.1)$ & $14(10.9)$ & $0.001^{*}$ & $108(5.1)$ & $26(7.6)$ & $20(14)$ & $<0.001^{*}$ \\
\hline TVR & $171(14.1)$ & $207(16.4)$ & $14(10.9)$ & 0.126 & $311(14.7)$ & $64(18.8)$ & $17(11.9)$ & 0.076 \\
\hline MACE & $204(16.9)$ & $293(23.1)$ & $68(53.1)$ & $<0.001^{*}$ & $383(18.1)$ & $92(27.1)$ & $90(62.9)$ & $<0.001^{*}$ \\
\hline
\end{tabular}

${ }^{*} p<0.05$ was considered significant; Mean values (standard deviation [SD]) and $n(\%)$ are reported for continuous and categorical variables, respectively; AVB - atrioventricular block; CPR - cardiopulmonary resuscitation; GI - gastrointestinal; IABP - intra-aortic balloon pump; MACE - major adverse cardiovascular event (cardiovascular death, reinfarction, TVR); TVR — target vessel revascularization; VT/VF - ventricular tachycardia/fibrillation; WBC-MPV - white blood cell-mean platelet volume; WMR - white blood cell count to mean platelet volume ratio

karyocytes are more sensitive than normal size platelets. Larger platelets are likely to be available well before the acute coronary artery occlusion responsible for ACS. For this reason, it is reasonable that hyperactive and larger platelets constitute a significant determinant of the thrombogenic process underlying the total occlusion of the coronary artery leading to STEMI. Several studies have suggested that MPV could be a marker of coronary perfusion in STEMI patients. Huczek et al. [18] showed MPV to be an independent predictor for the no-reflow phenomenon after pPCI. Estévez-Loureiro et al. [15] found that increased MPV is an independent predictor of both a patent IRA and 30-day mortality in patients with STEMI undergoing pPCI. RDW is a marker of variation in the size of circulating red cells (anisocytosis), and increased RDW levels can be considered the production of humoral mediators by the bone marrow. Inflammatory cytokines and neurohumoral mediators are activated in the process of STEMI. Elevated RDW was an independent predictor of mortality and morbidity in patients with heart disease. Tonelli et al. [19] found an independent relation between higher RDW levels and the risk of death and CV events in patients with CAD. Activated neutrophils release several proteolytic enzymes which increase the tissue destruction, such as acid phosphatase, myeloperoxidase, and elastase [20-22]. Activation of the neurohormonal 
Table 5. Cox-regression models revealing independent predictors of long-term cardiovascular mortality in all study patients.

\begin{tabular}{|c|c|c|c|c|}
\hline \multirow[t]{2}{*}{ Variables } & \multicolumn{2}{|l|}{ Model I } & \multicolumn{2}{|l|}{ Model II } \\
\hline & HR (95\% Cl) & $\mathbf{P}$ & HR (95\% CI) & $\mathbf{P}$ \\
\hline Age $\geq 70$ [years] & $1.726(1.298-2.294)$ & $<0.001^{*}$ & $1.800(1.354-2.393)$ & $<0.001^{*}$ \\
\hline Male gender & $0.725(0.534-0.984)$ & $0.039 *$ & $0.597(0.454-0.784)$ & $0.001 *$ \\
\hline Hypertension & $1.336(1.005-1.775)$ & $0.046^{*}$ & $1.427(1.086-1.876)$ & $0.011^{*}$ \\
\hline Diabetes mellitus & $1.440(1.061-1.955)$ & $0.019 *$ & $1.432(1.052-1.949)$ & $0.022^{*}$ \\
\hline Killip class $>1$ & $2.166(1.478-3.174)$ & $<0.001^{*}$ & $2.043(1.395-2.991)$ & $<0.001 *$ \\
\hline Heart rate $>100 \mathrm{bpm}$ & $2.646(1.714-4.085)$ & $<0.001^{*}$ & $2.349(1.539-3.584)$ & $<0.001^{*}$ \\
\hline Admission anemia & $1.407(1.064-1.862)$ & $0.017^{*}$ & $1.452(1.101-1.915)$ & $0.008 *$ \\
\hline RDW & 1.097 (1.016-1.183) & $0.018^{*}$ & $1.213(1.138-1.291)$ & $0.001 *$ \\
\hline \multicolumn{5}{|l|}{ PLR-NLR combination: } \\
\hline Low risk $(P L R \leq 162.0$ and NLR $\leq 6.32)$ & Reference & & Reference & \\
\hline Intermediate risk (PLR > 162.1 or NLR $>6.32)$ & $1.423(0.977-2.072)$ & 0.066 & $1.269(0.866-1.860)$ & 0.222 \\
\hline High risk (PLR $>162.0$ and NLR $>6.32)$ & $1.821(1.328-2.496)$ & $<0.001^{*}$ & $1.672(1.212-2.305)$ & $0.002^{*}$ \\
\hline \multicolumn{5}{|l|}{ WBC-MPV combination: } \\
\hline Low risk $(\mathrm{WBC} \leq 14,400$ and $\mathrm{MPV}>8.1)$ & Reference & & - & - \\
\hline Intermediate risk $(\mathrm{WBC}>14,400$ or $\mathrm{MPV} \leq 8.1)$ & $2.441(1.529-3.895)$ & $<0.001^{*}$ & - & - \\
\hline High risk (WBC $>14,400$ and $M P V \leq 8.1)$ & $5.375(3.230-8.947)$ & $<0.001^{*}$ & - & - \\
\hline \multicolumn{5}{|l|}{ WMR: } \\
\hline Low risk (WMR $\leq 1,653.47$ ) & - & - & Reference & \\
\hline Intermediate risk $(1,653.47<\mathrm{WMR} \leq 1,824.18)$ & - & - & $2.639(1.752-3.975)$ & $<0.001^{*}$ \\
\hline High risk (WMR > 1,824.18) & - & - & $7.075(4.869-10.280)$ & $<0.001^{*}$ \\
\hline Omnibus tests of model coefficients & \multicolumn{2}{|c|}{$\begin{array}{c}-2 \mathrm{LL}=2,675.6 \\
\chi^{2}=635.7, p<0.001\end{array}$} & \multicolumn{2}{|c|}{$\begin{array}{c}-2 \mathrm{LL}=2,564.8 \\
\chi^{2}=811.5, p<0.001\end{array}$} \\
\hline
\end{tabular}

${ }^{*} \mathrm{p}<0.05$ was considered significant; $\mathrm{Cl}$ - confidence interval; HR - hazard ratio; LL - Log Likelihhood; NLR - neutrophil-lymphocyte ratio; MPV - mean platelet volume; PLR - platelet-lymphocyte ratio; RDW — red blood cell distribution with; WBC — white blood cell; WMR - white blood cell count to mean platelet volume ratio

system, oxidative stress and inflammation in ACS increase the catecholamine levels and the plasma cortisol levels cause bone marrow suppression and down-regulation of the lymphocyte proliferation and differentiation with aggravated lymphocyte apoptosis. In a recent study, He et al. [23] showed that average NLR was a useful and powerful predictor of mortality and adverse-outcomes in Chinese patients presenting with STEMI. Elevated leukocytes are associated with increased mortality in patients with myocardial infarction [24]. Several mechanisms can explain this relationship; 1) leukocytes can cause injury of endothelial cells by oxidative and proteolytic damage, 2) leukocytes can plug the microvascularization, 3) leukocytes can stimulate hypercoagulability and activated monocytes [25]. In a recent study, Dharma et al. [25] reported that high blood leukocyte count on admission was an independent predictor of $\mathrm{CV}$ events in patients with NSTEMI. Furthermore, Sabatine et al. [26] found that an elevated baseline WBC count correlated with impaired myocardial perfusion and increased 6 -month mortality in patients with STEMI. Maden et al. [27] showed that higher WBC and MPV is associated with occluded IRA in patients with STEMI. Karahan et al. [28] observed that increased WBC and MPV are independent predictors of impaired microvascular perfusion in patients with STEMI. Increased WBCs may appear as a significant factor showing impaired microvascular reperfusion. Recently, Dehghani et al. [11] investigated a novel parameter called WMR as a marker predicting long-term outcomes in patients with NSTEMI. They suggested that WMR is a better predictor of worse outcomes in patients with NSTEMI than WBC and MPV [11]. In light of these evidences, we decided to evaluate the prognostic value of WMR with a large number of patients with STEMI undergoing pPCI and demonstrated that WMR is a better indicator of predicting the poor 


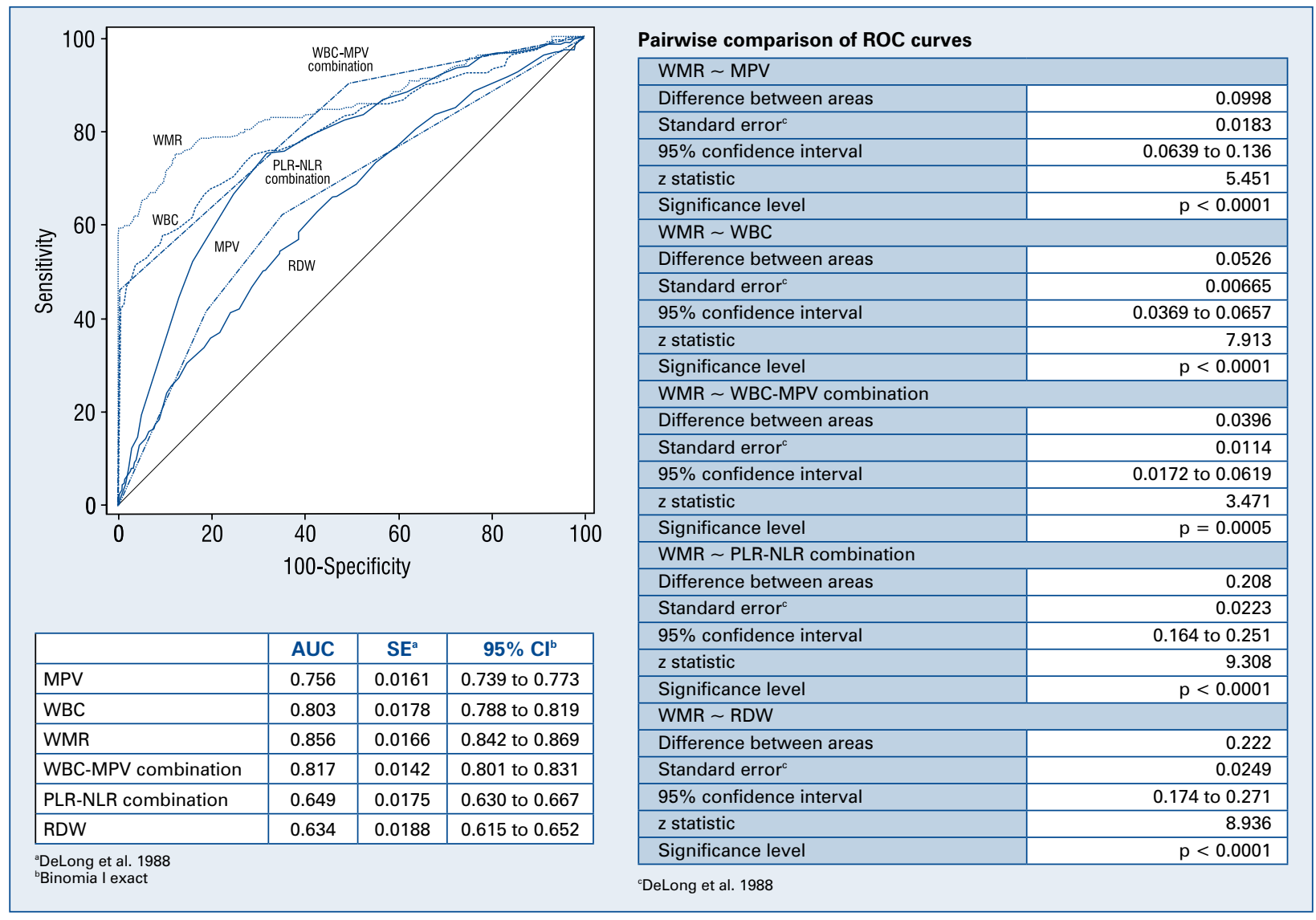

Figure 1. Receiver operating characteristic curves identifying the discrimination thresholds of white blood cell (WBC)-mean platelet volume (MPV) combination, white blood cell count to mean platelet volume ratio (WMR), platelet to lymphocyte ratio and neutrophil to lymphocyte ratio (PLR-NLR) combination and their components for long-term mortality; RDW — red blood cell distribution with.

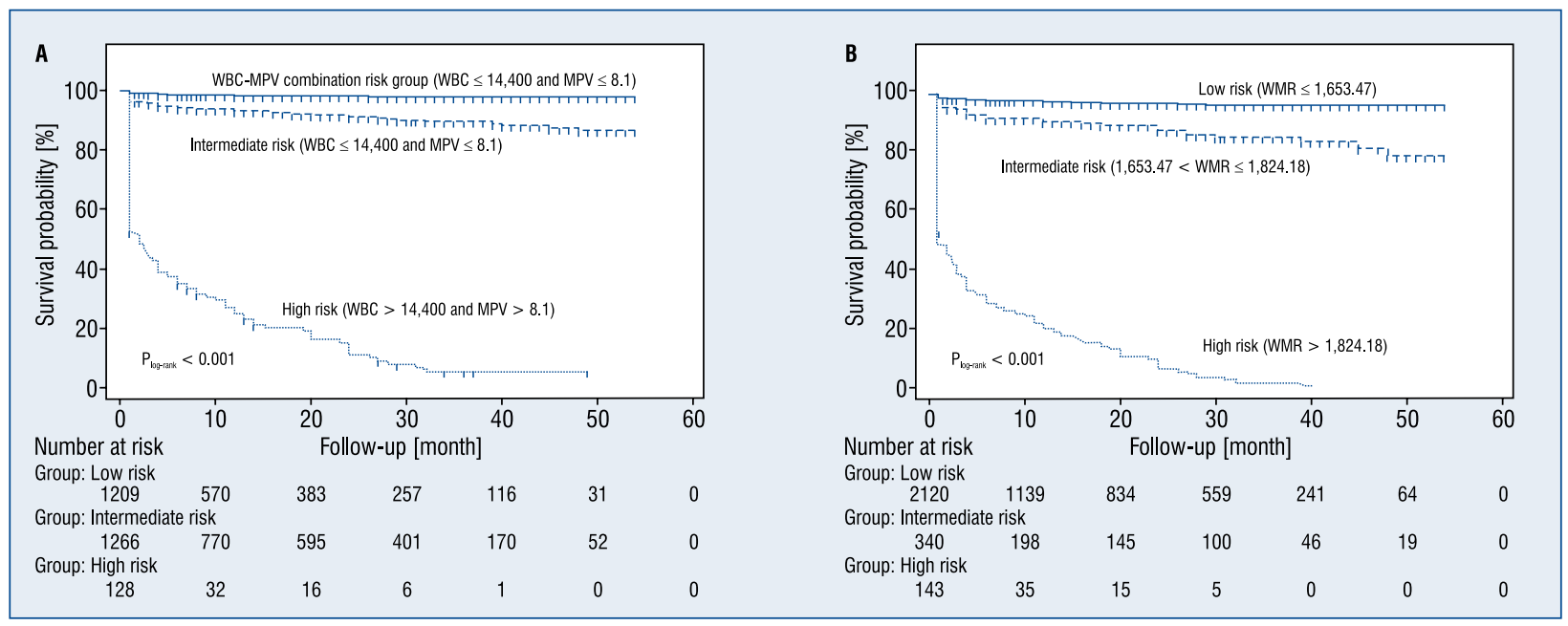

Figure 2. Kaplan-Meier cumulative survival curves for patients with mortality according to combined white blood cell (WBC) (A)-mean platelet volume (MPV) and white blood cell count to mean platelet volume ratio (WMR) (B) risk stratification. 
outcomes in STEMI than MPV, RDW, PLR-NLR and WBC-MPV combinations.

White blood cell count to mean platelet volume ratio has some key properties that a novel CV prognostic marker should have [29, 30]. First, WMR is obtained easily from routine complete blood counts without additional work or cost. Second, it provides information about hard endpoints such as in-hospital an long-term mortality. Moreover, it is also useful for risk stratification in patients classified into risk groups according to WMR levels. Such risk stratification may allow clinicians to determine patients who are at higher risk and individualizing the therapy. In patients with elevated WMR, more intensive medical therapy and more aggressive control of $\mathrm{CV}$ risk factors may be considered. In this high-risk population, more close follow-up visits can also be arranged. However, further studies with long-term follow-up and large-scale prospective data are needed to elucidate the exact role of WMR in patients with CAD.

\section{Limitations of the study}

Our study findings should be interpreted with some limitations. First, it was a single-center, retrospective study without randomization. Inflammatory markers, such as high-sensitivity $\mathrm{C}$-reactive protein, B-type natriuretic peptide, other pro-inflammatory cytokines, and markers of oxidative stress were not analyzed. Using a spot laboratory value of complete blood counts rather than values at a time-interval is another limitation of this study.

\section{Conclusions}

In conclusion, higher WMR value on admission was associated with worse outcomes in patients with STEMI and independently predicted the longterm mortality better than other complete blood count components, such as MPV, RDW, PLR-NLR and WBC-MPV combinations.

\section{Conflict of interest: None declared}

\section{References}

1. Libby P. What have we learned about the biology of atherosclerosis? The role of inflammation. Am J Cardiol, 2001; 88: 3J-6J.

2. Kannel WB, Anderson K, Wilson PW. White blood cell count and cardiovascular disease. Insights from the Framingham Study. JAMA, 1992; 267: 1253-1256.

3. Frossard M, Fuchs I, Leitner JM et al. Platelet function predicts myocardial damage in patients with acute myocardial infarction. Circulation, 2004; 110: 1392-1397.
4. Chung S, Song YB, Hahn JY et al. Impact of white blood cell count on myocardial salvage, infarct size, and clinical outcomes in patients undergoing primary percutaneous coronary intervention for ST-segment elevation myocardial infarction: A magnetic resonance imaging study. Int J Cardiovasc Imag, 2014; 30: 129-136.

5. Mikhailidis DP, Barradas MA, Mier A et al. Platelet function in patients admitted with a diagnosis of myocardial infarction. Angiology, 1987; 38: 36-45.

6. Tamura A, Watanabe T, Nasu M. Association between neutrophil counts on admission and left ventricular function in patients successfully treated with primary coronary angioplasty for first anterior wall acute myocardial infarction. Am J Cardiol, 2001; 88: 678-680.

7. Uyarel H, Ergelen M, Cicek G et al. Red cell distribution width as a novel prognostic marker in patients undergoing primary angioplasty for acute myocardial infarction. Coron Artery Dis, 2011; 22: 138-144.

8. Elbasan Z, Gur M, Sahin DY et al. Association of mean platelet volume and pre- and postinterventional flow with infarct-related artery in ST-segment elevation myocardial infarction. Angiology, 2013; 64: 440-446.

9. Sahin DY, Elbasan Z, Gur M et al. Neutrophil to lymphocyte ratio is associated with the severity of coronary artery disease in patients with ST-segment elevation myocardial infarction. Angiology, 2013; 64: 423-429.

10. Yayla C, Akboga MK, Canpolat U et al. Platelet to lymphocyte ratio can be a predictor of infarct-related artery patency in patients with ST-segment elevation myocardial infarction. Angiology, 2015; 66: 831-836.

11. Dehghani MR, Rezaei Y, Taghipour-Sani L. White blood cell count to mean platelet volume ratio as a novel non-invasive marker predicting long-term outcomes in patients with non-ST elevation acute coronary syndrome. Cardiol J, 2015; 22: 437-445.

12. Thygesen K, Alpert JS, Jaffe AS et al. Third universal definition of myocardial infarction. Eur Heart J, 2012; 33: 2551-2567.

13. Cicek G, Acikgoz SK, Bozbay M et al. Neutrophil-lymphocyte ratio and platelet-lymphocyte ratio combination can predict prognosis in patients with ST-segment elevation myocardial infarction undergoing primary percutaneous coronary intervention. Angiology, 2015; 66: 441-447.

14. Acet H, Ertas F, Akil MA et al. Novel predictors of infarct-related artery patency for ST-segment elevation myocardial infarction: Platelet-to-lymphocyte ratio, uric acid, and neutrophil-to-lymphocyte ratio. Anadolu Kardiyol Derg, 2015; 15: 648-656.

15. Estevez-Loureiro R, Salgado-Fernandez J, Marzoa-Rivas R et al. Mean platelet volume predicts patency of the infarct-related artery before mechanical reperfusion and short-term mortality in patients with ST-segment elevation myocardial infarction undergoing primary percutaneous coronary intervention. Thromb Res, 2009; 124: 536-540.

16. Azab B, Shah N, Akerman M, McGinn JT, Jr. Value of platelet/ lymphocyte ratio as a predictor of all-cause mortality after nonST-elevation myocardial infarction. J Thromb Thrombolysis, 2012; 34: 326-334.

17. Lopez-Cuenca AA, Tello-Montoliu A, Roldan V, Perez-Berbel P, Valdes M, Marin F. Prognostic value of mean platelet volume in patients with non-ST-elevation acute coronary syndrome. Angiology, 2012; 63: 241-244.

18. Huczek Z, Kochman J, Filipiak KJ et al. Mean platelet volume on admission predicts impaired reperfusion and long-term mortality in acute myocardial infarction treated with primary percutaneous coronary intervention. J Am Coll Cardiol, 2005; 46: 284-290.

19. Tonelli M, Sacks F, Arnold M et al. Relation between red blood cell distribution width and cardiovascular event rate in people with coronary disease. Circulation, 2008; 117: 163-168. 
20. Reichlin T, Socrates T, Egli P et al. Use of myeloperoxidase for risk stratification in acute heart failure. Clin Chem, 2010; 56: 944-951.

21. Tousoulis D, Antoniades C, Koumallos N, Stefanadis C. Pro-inflammatory cytokines in acute coronary syndromes: from bench to bedside. Cytokine Growth Factor Rev, 2006; 17: 225-233.

22. Mehta J, Dinerman J, Mehta $P$ et al. Neutrophil function in ischemic heart disease. Circulation, 1989; 79: 549-556.

23. He J, Li J, Wang Y, Hao P, Hua Q. Neutrophil-to-lymphocyte ratio (NLR) predicts mortality and adverse-outcomes after ST-segment elevation myocardial infarction in Chinese people. Int J Clin Exp Pathol, 2014; 7: 4045-4056.

24. Menon V, Lessard D, YarzebskiJ, Furman MI, GoreJM, Goldberg RJ. Leukocytosis and adverse hospital outcomes after acute myocardial infarction. Am J Cardiol, 2003; 92: 368-372.

25. Dharma S, Hapsari R, Siswanto BB, van der Laarse A, Jukema JW. Blood leukocyte count on admission predicts cardiovascular events in patients with acute non-ST elevation myocardial infarction. Int J Angiol, 2015; 24: 127-132.

26. Sabatine MS, Morrow DA, Cannon CP et al. Relationship between baseline white blood cell count and degree of coronary artery disease and mortality in patients with acute coronary syndromes: A TACTICS-TIMI 18 (Treat Angina with Aggrastat and determine Cost of Therapy with an Invasive or Conservative Strategy-Thrombolysis in Myocardial Infarction 18 trial) substudy. J Am Coll Cardiol, 2002; 40: 1761-1768.

27. Maden O, Kacmaz F, Selcuk H et al. Relationship of admission hematological indexes with myocardial reperfusion abnormalities in acute ST segment elevation myocardial infarction patients treated with primary percutaneous coronary interventions. Can J Cardiol, 2009; 25: e164-e168.

28. Karahan Z, Ucaman B, Ulug AV et al. Effect of hematologic parameters on microvascular reperfusion in patients with ST-segment elevation myocardial infarction treated with primary percutaneous coronary intervention. Angiology 2016; 67: 151-156.

29. Hlatky MA, Greenland P, Arnett DK et al. Criteria for evaluation of novel markers of cardiovascular risk: A scientific statement from the American Heart Association. Circulation, 2009; 119: 2408-2416.

30. Morrow DA, de Lemos JA. Benchmarks for the assessment of novel cardiovascular biomarkers. Circulation, 2007; 115: 949-952. 